\title{
COMERCIALIZACIÓN DE MIEL EN EL MUNICIPIO DE ESPITA, YUCATÁN
}

\section{HONEY MARKETING IN THE MUNICIPALITY ESPITA, YUCATAN}

\begin{tabular}{r|c|l} 
Manuel J. Caro Encalada & Carlos E. Leyva Morales & Maricarmen Chi Tuz \\
Facultad de Economía, & Facultad de Economía, & Facultad de Economía, \\
Universidad Autónoma de Yucatán & Universidad Autónoma de Yucatán & Universidad Autónoma de Yucatán \\
Correo: mcencala@hotmail.com & Correo: clmoral@tunku.uady.mx & Correo: maika_44mar@hotmail.com
\end{tabular}

\section{RESUMEN}

El estudio buscó analizar la relación entre los agentes comerciales que participan en la comercialización de miel en el municipio de Espita, Yucatán y el poder de negociación y de ganancia comercial que se obtiene en esta actividad productiva. El trabajo se basó en un diseño de comprobación del tipo transeccional en su variante correlacional aplicando las técnicas de revisión de literatura, encuesta a apicultores, censo a centros de acopio y entrevista a informantes clave. El análisis de los resultados se fundamentó en la teoría de la comercialización de Caldentey (2004) y en la teoría del mercado propuesta por Stamer (1999) y Haag y Soto (1984). Se concluye que la relación analizada es desventajosa para el apicultor, debido a que la estructura oligopsónica de los centros de acopio que intervienen en el proceso de comercialización de miel en el municipio de Espita da como consecuencia que el poder de fijación del precio de compra lo tengan estos intermediarios comerciales, y con ello el poder de negociación, y que la comercialización de la miel no sea un proceso dinámico ni beneficioso para el productor, este tiene el mayor gasto de recursos, tiempo y trabajo en la producción mientras que los intermediarios solo compran y movilizan en tiempos cortos la miel hacia sus empresas 
matrices llevándose la mayor ganancia comercial sin realizar tanto esfuerzo y riesgo.

Palabras clave: Comercialización, miel, Espita.

Clasificación JEL: Q1, Q13.

\section{ABSTRACT}

The study seeking to analyze the relationship between commercial actors involved in the marketing of honey in the municipality of Espita, Yucatán and the power of negotiation and commercial gain obtained in this productive activity. The work was based on a design of transectional type checking in its correlational variant applying the techniques of literature review, survey of beekeepers, census to Collection Centers and key informants interviewing. The analysis of the results is based in the commercialization theory of Caldentey (2004) and in the theory of the market proposed by Stamer (1999) and Haag and Soto (1984). Concludes that the analyzed relationship is disadvantageous for the beekeeper, since the structure oligopsonica of the collection centers that are involved in the process of commercialization of honey in the municipality of Espita gives as result that the power of the purchase price fixing it to these commercial intermediaries, and thereby the bargaining power, and that the marketing of honey is not a dynamic process or beneficial for the producer, this has the greater expenditure of resources, time and labor in production while intermediaries only buy and mobilize in short times honey into their parent companies carrying the greater commercial profit without making much effort and risk.

Keywords: Marketing, honey, Espita.

JEL Clasification: Q1, Q13

\section{INTRODUCCIÓN}

La producción apícola de México es una actividad que tiene presencia en todo el país, actualmente ocupa el sexto lugar a nivel internacional en cuanto a producción (los principales países con los que compite son: China, Estados Unidos y Argentina) y el tercer lugar en el rubro exportador, solo después de China y Argentina (El Economista, 2011). Asimismo, por su generación de ingreso de divisas se le ubica como una de las tres actividades más importantes en el sector pecuario nacional, las dos primeras son la ganadería bovina y la producción de camarón (SIAP, 2010).

Los canales de comercialización por los que transita la producción de miel en el mercado interno de México son dos básicamente. El primero se da mediante la venta directa de los productores al consumidor, que envasan la producción y efectúan la venta en su región. En tanto el segundo canal de comercialización se da con la industria, la que utiliza la miel como un ingrediente para la elaboración de alimentos como: cereales, yogurts, dulces y panes; o bien, como materia prima para la industria tabacalera y cosmetológica. Aproximadamente 7,420 toneladas de miel en el 2008 fueron comercializadas directamente por los productores en cubetas de 20 litros o en frascos de presentación individual, en tanto que 6,904 toneladas fueron consumidas por la industria cosmetológica, tabacalera y dulcera, otras 8,115 toneladas fueron distribuidas a la industria alimenticia y restaurantera y 7,600 toneladas fueron envasadas por las diez grandes empresas y 82 pequeñas que se encuentran distribuidas en toda la República Mexicana (Aserca, 2010).

Dentro de la república mexicana, la península de Yucatán es considerada como la zona de mayor producción de miel en el país, su producción se da durante los meses de diciembre a junio debido a la multiflora que posee dicha región. Según datos de la SAGARPA (2009) la península de Yucatán suma el $29.2 \%$ del volumen total producido en el país, lo cual 
demuestra la importancia de ésta región como la principal productora de miel en México.

La producción de miel de la península de Yucatán ha mantenido en términos generales una tendencia cíclica (de altas y bajas) en su comportamiento registrando en el año 2008 su nivel más alto de producción, de aproximadamente 20,779 toneladas, aunque en el 2009 esta tendencia se revirtió (Cuadro 1), alcanzándose una producción de solo poco más de quince mil toneladas, debido esto último a las bajas temperaturas y la sequía que imperó en ese año.

Cuadro 1.

\begin{tabular}{|l|c|c|c|c|c|c|c|c|c|}
\hline \multicolumn{7}{|c|}{ Participación Estatal en la Producción de Miel en México (Toneladas) } \\
\hline & $\mathbf{2 0 0 1}$ & $\mathbf{2 0 0 2}$ & $\mathbf{2 0 0 3}$ & $\mathbf{2 0 0 4}$ & $\mathbf{2 0 0 5}$ & $\mathbf{2 0 0 6}$ & $\mathbf{2 0 0 7}$ & $\mathbf{2 0 0 8}$ & $\mathbf{2 0 0 9}$ \\
\hline Campeche & 8,521 & 8,047 & 6,412 & 5,323 & 5,853 & 6,016 & 8,206 & 8,817 & 6,976 \\
\hline $\begin{array}{l}\text { Quintana } \\
\text { Roo }\end{array}$ & 2,544 & 2,486 & 2,504 & 2,573 & 1,832 & 2,640 & 2,333 & 2,188 & 2,170 \\
\hline Yucatán & 9,169 & 10,020 & 8,427 & 9,375 & 6,644 & 8,417 & 8,483 & 9,774 & 6,244 \\
\hline Total & 20,234 & 20,553 & 17,343 & 17,271 & 14,329 & 17,073 & 19,022 & 20,779 & 15,390 \\
\hline
\end{tabular}

Fuente: Servicio de Información Agroalimentaria y Pesquera / SAGARPA 2009

De los tres estados que conforman la península, el de Yucatán es el principal productor y exportador de miel a nivel nacional, esto último lo realiza principalmente a países como Alemania, España, Suiza, Italia, Inglaterra, Francia, Estados Unidos, Canadá y Arabia Saudita, con ganancias de mil 500 a 3, 000 millones de pesos anuales (El Financiero, 2009).

En el 2008 del total regional Campeche aportaba el $42 \%$ del volumen de miel producido ocupando el segundo puesto después de Yucatán (con el $47 \%$ ); en tanto que Quintana Roo sólo aportaba el $11 \%$ de este total (SAGARPA, 2009).
La actividad apícola se encuentra extendida por todo el Estado y cumple una importante función socioeconómica ya que genera empleos y capta divisas para la entidad. A este último respecto, las cifras disponibles indican que entre los años 2003 a 2005, los volúmenes de la miel yucateca exportada (envasada en tambores de $300 \mathrm{Kg}$ ) descendieron, pasaron de 4, $393,048 \mathrm{Kg}$ en el 2003, a 2, 435,796 Kg en el año 2004 y 2, 096,776 Kg en el 2005, esto causado principalmente por los cambios climáticos en la región como: huracanes, granizadas, frío y nublados (Medina, 2006). Sin embargo, de la producción local de miel del estado de Yucatán, que promedia entre ocho y diez mil toneladas anuales, el 80 por ciento se vende al extranjero, el 15 por ciento se destina al mercado nacional y el cinco por ciento se consume en la entidad (SAGARPA, 2009). Por ello, la comercialización al exterior de la miel de Yucatán aporta al estado el segundo mayor ingreso por concepto de divisas del sector agropecuario, el primero le corresponde a las exportaciones de pescados y mariscos (Secretaria de Fomento Económico, 2010).

Sin embargo, no obstante que el estado de Yucatán es una de las entidades con mayor nivel de producción de miel en el país, enfrenta diversos problemas técnico-económicos como: la llegada de la abeja africana (Güemez, 2002); la presencia del ácaro Varroa Jacobsoni Oudemans (Güemez, 2003), los efectos climáticos (Villanueva, 1996), la competencia y bajos precios de la miel durante años en el mercado internacional, los bajos precios pagados a los apicultores y su limitada variación cuando estos se integran en otros mercados (Aserca, 2010), la poca inversión en los apiarios, la baja rentabilidad debido a fallas tecnológicas en el proceso productivo de esta actividad, la falta de organización de los productores para elevar sus niveles de productividad y mejorar sus condiciones para producir con mayor calidad, certificación y diversificación de productos (Magaña, 2007), y el problema del intermediarismo; que en conjunto frenan la actividad y no permiten un mejor desarrollo de ésta.

Bajo este contexto, el objetivo del presente estudio se orientó a analizar la 
relación entre los agentes comerciales que participan en la comercialización de miel en el municipio de Espita, Yucatán y el poder de negociación y de ganancia comercial que se obtiene en esta actividad productiva.

\section{METODOLOGÍA}

El trabajo se basó en un diseño de comprobación del tipo transeccional en su variante correlacional (Hernández et al., 2010), siendo su propósito el establecer la relación existente entre las variables consideradas, analizando su incidencia y los valores en que se manifiestan.

Se realizó una revisión de literatura, la cual abarcó la recopilación de información de primera mano proveniente de documentos y fuentes electrónicas de diversas dependencias nacionales y estatales relacionadas con la actividad apícola (SIAP, SAGARPA, Secretaría de Fomento Agropecuario y Pesquero, etc.), así como las provenientes de trabajos de campo realizados sobre el tema. El instrumento utilizado fue una bitácora de trabajo donde se registró cada una de las referencias seleccionadas.

El trabajo de campo se basó en una encuesta, en donde se aplicó un muestreo aleatorio al azar simple, cuyo universo poblacional se basó en la lista de los apicultores de la localidad estudiada (Espita), la cual fue seleccionada por estar comprendida en la zona Oriente, una de las dos zonas productoras de miel de mayor importancia en el estado (junto con la zona Sur aportan actualmente el $70 \%$ de la producción total del estado). El tamaño de muestra que se calculó fue de 62 apicultores. Para el acopio de información se diseñó una cédula de entrevista, siendo la información que se recopiló la siguiente: tipo de propiedad del apiario, tamaño, volumen de producción, equipo con que cuenta, estrategias para la venta de sus productos, forma como determinan sus precios de venta, quién determina el precio de venta, temporada en que se vende la miel y sus otros productos, los agentes comerciales a quienes venden, los ingresos que perciben por la venta de sus productos así como los principales problemas que enfrentan en la comercialización. La cédula constó de 72 preguntas de las cuales 55 fueron preguntas cerradas y 17 abiertas.

Se efectuó también la aplicación de un censo a los cinco centros de acopio existentes en el municipio. La información que se recopiló de estos fue principalmente la siguiente: tamaño del centro, capacidad instalada, infraestructura con que cuenta, tipo de centro que es, estrategias que llevan a cabo para la compra y venta de los productos que acopian, forma como determinan sus precios de venta, quién determina el precio de venta, temporada en que se vende la miel y los otros productos que acopian, agentes comerciales a quienes venden, ingresos que perciben por la venta de sus productos así como los principales problemas que enfrentan en la comercialización. La cédula censal que se aplicó constó de 44 preguntas en total, de las cuales 39 fueron preguntas cerradas y cinco abiertas.

Por último, la información de los informantes clave se obtuvo mediante la aplicación de una entrevista dirigida a las personas de importancia en el ámbito agrícola en la municipalidad: el presidente de la Asociación Ganadera Local de Espita y el presidente municipal de la misma; para lo cual se diseñó una guía de entrevista que constó de diez preguntas abiertas en donde se cuestionó sobre: la demanda de miel, la red de caminos, los precios, los problemas de la comercialización y los apoyos a la misma en el municipio, entre otros aspectos de interés.

Los datos tanto de los apicultores como de acopiadores se capturaron en una base de datos en el programa Windows Excel en una hoja de codificación en la cual se registraron las respuestas a las preguntas realizadas, siendo los principales estadísticos obtenidos: porcentajes, valores promedio, sumas y restas. En tanto que la proveniente de los informantes clave se sistematizó mediante una hoja de análisis de contenido.

El análisis de los resultados del trabajo se fundamentó en la teoría de la comercialización propuesta por Caldentey (2004) acerca de los agentes omerciales que integran los canales de comercialización de esta actividad, los márgenes comerciales, las estrategias comerciales, los precios de com- 
pra y venta de la miel y las funciones comerciales (acopio, preparación para el consumo y distribución); y en la teoría del mercado propuesta por Stamer (1999) y Haag y Soto (1984), la cual considera el tipo de mercado en que se encuentra inserta la actividad apícola, la oferta y precio de la miel, así como los factores que determinan el comportamiento de estas variables económicas en el mercado de este producto.

\section{RESULTADOS}

El municipio de Espita está localizado al Oriente del Estado de Yucatán, aproximadamente a $152 \mathrm{~km}$ de la ciudad de Mérida, capital del Estado y a $28 \mathrm{~km}$ de la ciudad de Tizimín (Figura 1). Limita al norte con el municipio de Sucilá, al sur con Tinum y Uayma, al este con Temozón y Calotmul y al oeste con Cenotillo y Dzitas. Esta comprendido entre los paralelos $20^{\circ} 53^{\prime}$ y $21^{\circ} 08^{\prime}$ latitud norte y los meridianos $88^{\circ} 16^{\prime}$ y $88^{\circ} 27^{\prime}$ de longitud oeste; y tiene una altura de 20 metros sobre el nivel del mar (Gobierno del estado de Yucatán, 2010).

\section{Figura 1.}

Ubicación geográfica de Espita

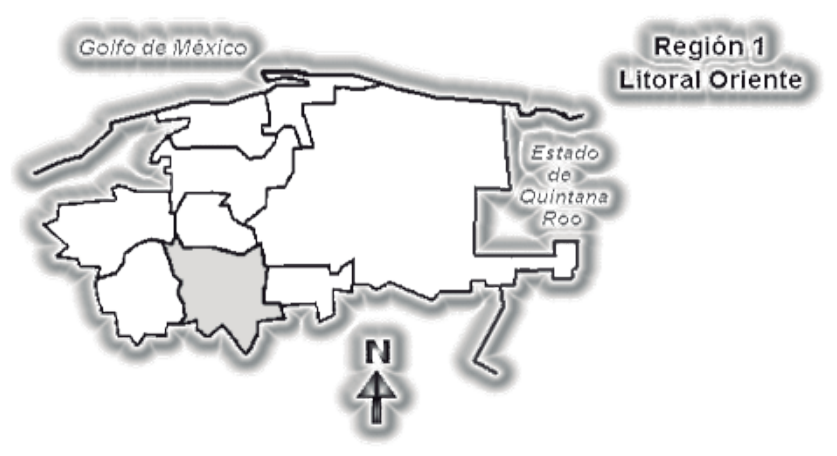

Revista de Economía - Vol. XXVIII - Núm. 78
Espita "Lugar de Poca Agua" tiene como actividad principal la agrícola El municipio está conformado por las siguientes comisarías: Holca, Kunche, Nacuche, San Pedro Chenchela, Tuzik, Xualtez, Xuilub y Santa Cruz Regadillo.

Presenta un clima semi-seco y cálido con lluvias en verano, una temperatura media anual de $24^{\circ} \mathrm{C}$ y $26^{\circ} \mathrm{C}$, su precipitación pluvial va de menos 600 a $800 \mathrm{~mm}$, los vientos dominantes son en dirección sureste y noroeste y tiene una superficie total de $717.48 \mathrm{~km} 2$ que representa el $1.45 \%$ del total del estado (Gobierno del estado de Yucatán, 2010).

La población económicamente activa del municipio asciende a 3,850 personas, de las cuales 3,829 se encuentran ocupadas y se encuentran distribuidas por sector económico de la siguiente manera (Grafica 1):

Gráfica 1.

Porcentaje de la población ocupada según sector de actividad

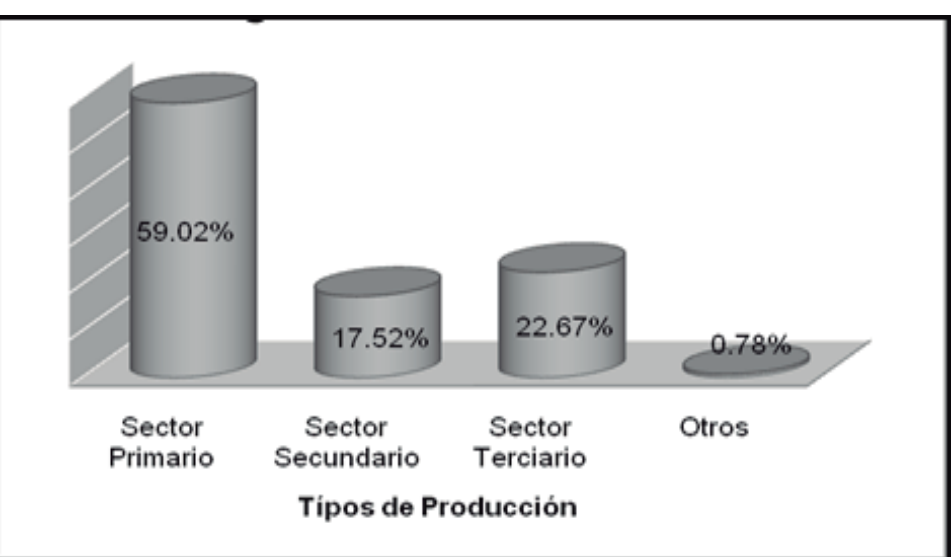

Fuente: Plan municipal de desarrollo rural sustentable de Espita municipio del estado de Yucatán, 2008. 
Como se aprecia, la actividad económica preponderante de la localidad es la actividad primaria, principalmente actividades pecuarias como forma de autoempleo de la familia de los pobladores de la municipalidad, en donde cuando los animales alcanzan el peso adecuado los ofrecen en venta a intermediarios o personas que estén interesadas en su consumo o comercio.

Dentro de los tres sectores económicos las actividades principales que realizan los habitantes de Espita son: agricultura, bovinocultura, porcicultura, avicultura, apicultura, elaboración de artesanías típicas de la región, pequeño comercio y la prestación de servicios básicos como el transporte.

\subsection{Características Generales de los Apicultores de Espita}

Los apicultores que existen en total en Espita son 312 quienes son personas de edad avanzada. Estos se encuentran distribuidos en tres estratos:

\section{De 1-20 Colmenas}

\section{De 21-50 Colmenas}

De 50 Colmenas en adelante.

El primer estrato lo conforman el $45.9 \%$ de los apicultores del municipio, el segundo el $40.5 \%$ en tanto que el tercer estrato solo comprende al $13.5 \%$ de estos, por lo que se puede decir que la mayoría de los apicultores de esta municipalidad son de pequeños a medianos.

La apicultura es realizada en el municipio desde hace años y se ha transmitido de generación en generación a lo largo del tiempo. Esta les sirve como medio de subsistencia a los habitantes de la municipalidad, los apoya en sus ingresos (como autoconsumo) y gastos (como pago en espe- cie a otros) y les ofrece asimismo un ingreso extra que combinan con el obtenido de sus otras actividades (agricultura, cría de animales de traspatio, ganadería y en algunos casos, el comercio) para así solventar los gastos cotidianos de sus familias.

La variabilidad que han presentado los apicultores en cuanto al número de sus colmenas se caracterizó porque solo en el 13.57 \% de éstos se ha mantenido el mismo número de colmenas en sus apiarios desde su inicio en la actividad, en tanto que en el $86.43 \%$ si ha variado el número de éstas en casi su totalidad, generalmente en la forma de un decremento de las mismas. Esta variabilidad negativa la asocian a los cambios climáticos y biológicos que se han venido dando en el estado a lo largo de los últimos años, como: sequías, huracanes, la varroa, los ataques de insectos a las colonias de los apicultores y la africanización de las abejas; aunque en la actualidad consideran que éste número se está recuperando.

Los apicultores de Espita tienen experiencia en el manejo de esta actividad y los procedimientos que realizan al momento de extraer la miel se caracterizan por: ser efectuados de manera manual, emplear muy poco conocimiento de la tecnología existente para este proceso productivo, y basarse en una muy baja preparación técnica del productor, generalmente ésta es poca a casi nula.

Estos productores están ubicados en su gran mayoría en terrenos ejidales y las actividades que usualmente realizan en su apiario son: limpieza, revisión, alimentación, aplicación de medicamentos, mantenimiento de apiario, la cosecha de miel y cera, el transporte del equipo de cosecha de miel y el transporte de agua. El apicultor es quien mayormente realiza cas todas las tareas anteriores, a excepción de las cosechas de miel y cera, en donde es auxiliado por sus hijos y trabajadores eventuales principalmente. Los períodos del año en que realizan las tareas señaladas son: limpieza, de mayo a noviembre; revisión, todo el año; alimentación, de agosto a octubre; aplicación de medicamentos, de agosto a octubre; mantenimiento, en enero principalmente; cosecha de miel, cera, y transporte de equipo de cosecha de miel, de enero a abril; transporte de agua, todo el año y la producción de reinas, de mayo a julio.

\section{Enero a Junio de 2012 - Págs: 9-35}


En tanto que las actividades que más demandan del tiempo del apicultor en el año son: limpieza del apiario, la revisión y el transporte del agua.

La infraestructura que poseen en sus apiarios también es muy poca, así como los conocimientos que tienen del manejo de ésta, esto provoca que existan en cada ciclo productivo pérdidas en el desarrollo de su proceso de producción.

En lo que respecta a los principales productos de la colmena que obtienen estos apicultores, el $99 \%$ produce miel y cera, mientras que solo un uno por ciento produce jalea real. De los que producen cera, el $80 \%$ la venden (promedio de producción total por temporada de $286.5 \mathrm{Kg}$. de cera), siendo el precio promedio que recibieron de la venta en el año 2008 de $\$ 29.41$, lo anterior dio como resultado que el ingreso promedio por la venta de cera en esa temporada fuera de $\$ 8,426.00$. El $20 \%$ restante que la producen, no la venden porque consideran que el procedimiento para la elaboración de ésta es muy cansado y el precio que se paga en el mercado muy bajo, razones por las que deciden quedarse con ella y utilizarla para alimentar a sus abejas o bien para realizar las quemas cuando van a alimentarlas, de esta manera no compran aceite vegetal para tal fin.

\subsection{Aspectos del Mercadeo de Miel en Espita}

En Espita el 95 \% de la producción de miel que se obtiene es destinada a la venta en los centros de acopio existentes, en tanto que el $5 \%$ restante se la quedan los apicultores, de esta, el $4 \%$ es para autoconsumo y el $1 \%$ es para la venta directa al consumidor final.

El precio que recibieron los apicultores en la temporada analizada fue de $\$ 30.00$ por kilogramo en promedio de los acopiadores, este se debió principalmente por la escasez general que hubo de miel en Yucatán en el año 2008, consecuencia de la sequía que se dio en toda la entidad 1 . No obstante lo anterior, fue el año en el que el precio de la miel fue mejor pagado en todo el estado ya que en años anteriores el precio había oscilado entre los $\$ 20.00$ y $\$ 10.00$ por $\mathrm{kg}$.

En la Gráfica 2 a continuación se presenta la variación de los precios de la miel en 2008 en el municipio, se puede observar que al inicio de la temporada de producción fue cuando se pagó el mejor precio, entre los $\$ 30.00$ a $\$ 35.00$ el kilogramo durante los meses de enero a mayo, en tanto que a partir del mes de junio este empezó a bajar llegando a venderse el kilogramo hasta en $\$ 18.00$.

Esto último tiene su explicación bajo los principios de la teoría económica en que estos meses son los más lluviosos en la región donde se ubica el municipio, lo cual ocasiona que la miel se humedezca y pierda valor de venta en el mercado por la reducción de su calidad (economía externa), además de que también en estos meses fue cuando se registró un incremento en la oferta de miel en la zona ocasionando, por las características oligopsónicas del mercado, la reducción en el precio pagado al productor.

En los últimos meses del ciclo productivo considerado (mayo a julio) este precio bajo más debido a los altos

En los últimos meses del ciclo productivo considerado (mayo a julio)
grados de humedad que presentó la miel cosechada en ese lapso. 
Gráfica 2.

Evolución de los precios de la miel en Espita

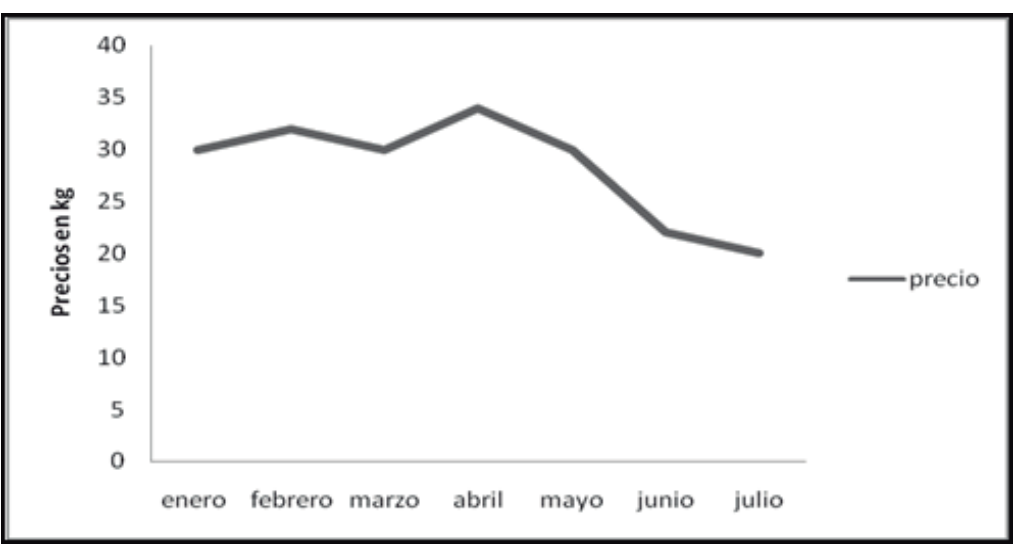

Fuente: Elaborado por el autor con la información de campo.

En cuanto a la forma de pago bajo la cual realizan la venta de miel los apicultores, ésta es principalmente en la forma de una parte al contado y la otra a crédito $(61.11 \%)$, siguiéndole en importancia el pago de contado (27.77\%).

El traslado de miel para la venta lo realizan mediante el uso de los siguientes medios de transporte: bicicleta $10.81 \%$, vehículo automotriz $67.56 \%$ (de estos el $29 \%$ es propio y el $71 \%$ es rentado) y triciclo 21.62 $\%$ (en las tres modalidades emplean galones para llevar a vender su miel al mercado). En tanto que el precio que pagan por transportar la miel en vehículo automotriz depende de cuán alejado esté el apiario del lugar en donde se realiza la venta del endulzante, en promedio el precio que pagaron fue de 230.55 pesos, siendo el más caro de 400 y el más barato de 150 pesos.

\subsection{Características de los Centros de Acopio}

Los principales centros de acopio a quienes los apicultores venden su miel en la municipalidad son: Miel Izamal, Apícola Maya, Miel Oaxaca y Miel Mex (Gráfica 3). Del total que se comercializa, el $38 \%$ de la miel es vendida al centro de acopio de la empresa Miel Mex, el $21 \%$ se vende al centro de Apícola Maya, el $34 \%$ al de Miel Izamal y el $7 \%$ restante al centro de la empresa denominada Miel Oaxaca. Los centros de acopio que son más conocidos y utilizados por los apicultores de la región son Miel Izamal, Apícola Maya y Miel Mex.

Gráfica 3.

Centro de Acopio a los que venden los apicultores de Espita

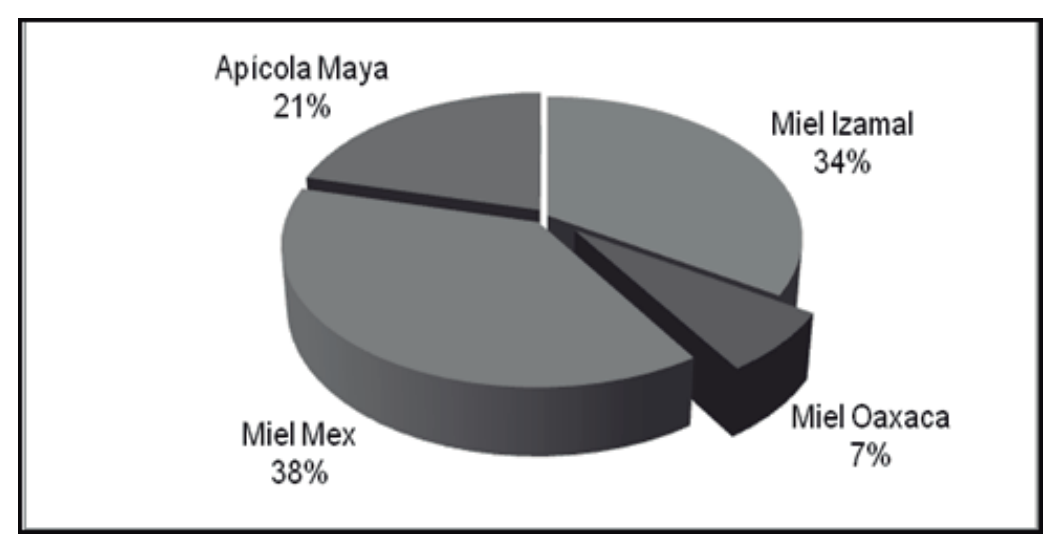

Fuente: Elaborado por el autor con la información de campo

Miel Izamal y Miel Mex se caracterizan porque captan miel durante todos los meses del año en el municipio, en tanto que Apícola Maya capta miel generalmente solo entre los meses de enero a junio.

Enero a Junio de 2012 - Págs: 9-35 
El precio más alto que pagaron estos centros, se dio en el mes de abril (\$35.00), ya que es cuando se recolecta la mejor miel que se produce en la municipalidad, al ser esta aromática, poseer menos humedad y ser más pura (es obtenida de la floración del Tzitzilché [Gymnopodium floribundum]). Este precio fue alto en relación a los de años anteriores debido a la escasez del producto que se dio como resultado de la sequía que se registró en la entidad. En contraparte, los meses en que se pagaron los precios más bajos por estas empresas fueron: mayo, debido a que es cuando existe un exceso de multiflora en la zona, y los de junio y julio ya que en estos meses es cuando se registra la mayor humedad en la miel producida, ocasionando las economías externas señaladas más arriba. Estos fueron de entre $\$ 20.00$ a $\$ 18.00$.

En Espita los cuatro centros de acopio son los que determinan el precio de compra de la miel que se comercializa, lo cual es resultado de que entre ellos detentan una estructura denominada en la literatura económica como oligopsonio de este producto en el municipio y resultan los más beneficiados en el comercio de este. Esto significa que el precio que se paga al apicultor en el municipio responde a un precio previamente fijado por estos agentes comerciales y no a las condiciones de la oferta que privan en el mercado, como marca la literatura económica bajo condiciones de libre competencia. Dando esto como resultado que los ingresos que obtiene el apicultor local sean menores, por ser poco su poder de negociación en la formación del precio al que se vende la miel, y que la mayor parte de los ingresos que genera esta actividad sean apropiados por estos agentes.

Estos centros se caracterizan por trabajar en asociación con alguna de las grandes empresas mayoristas comercializadoras que existen en el estado y la cantidad promedio de miel que reúnen es de 100 a 150 tambores, esto depende de la temporada de cosecha.
El precio promedio de compra 2 que registraron durante el año 2008 fue de $\$ 30.00$ por kilogramo y en su fijación intervino como principal factor el grado de humedad que tenga la miel, mientras más húmeda el precio resulta más bajo (criterio de calidad). En tanto que el precio promedio de venta que registraron fue de $\$ 32.00$ por kilogramo.

En lo que respecta a la infraestructura con la que cuentan los centros de acopio, básicamente está conformada por: báscula, refractómetro (instrumento para medir la humedad), tambores metálicos (que les proporciona la matriz) y el local donde operan; todos los centros no cuentan con medio de transporte propio dado que estos son proporcionados por la matriz con la que trabajan.

Los costos en que inciden generalmente durante el ciclo de recolección corresponden principalmente a los rubros de pagos de agua, luz y de mano de obra fija y eventual 3 (el único centro que registró gasto por concepto de pago de gasolina es de Miel Izamal). El valor promedio del costo total por mes de estos centros de acopio en el 2008 se desglosa en el cuadro siguiente:

Cuadro 2.

\begin{tabular}{|c|c|}
\hline \multicolumn{2}{|c|}{$\begin{array}{l}\text { Costos de Operación Promedio Mensual de lo } \\
\text { Centros de Acopio de Miel en Espita, Yucatár }\end{array}$} \\
\hline CONCEPTO & COSTO \\
\hline Transporte & $\$ 1,000.00$ \\
\hline Agua & $\$ \quad 200.00$ \\
\hline Luz & $\$ 100.00$ \\
\hline Mano de Obra & $\$ 6,000.00$ \\
\hline Mano de Obra Eventual & $\$ 1,000.00$ \\
\hline Limpieza & $\$ \quad 200.00$ \\
\hline TOTAL & $\$ 8,500.00$ \\
\hline
\end{tabular}

Fuente: Elaborado por el autor con la información de campo.

'El centro de acopio de Miel Oaxaca, que no cuenta con una buena ubicación en el municipio (está alejado del mismo) es el que ofrece el precio más bajo en el mercado local.

La mano de obra eventual es utilizada cuando se va a transportar la miel, se contrata a personas para que ayuden a subir los tambores al tríler en que se moviliza el producto. 
Los recursos económicos con que los centros de acopio de las empresas Miel Mex y Apícola Maya adquieren miel y cera son parcialmente de los encargados de estos, mientras que en el caso del centro de Miel Izamal, los recursos con que realiza sus adquisiciones son totalmente de la empresa matriz. La forma como estos centros realizan el pago del producto que les ofrecen los apicultores es: Miel Mex y Apícola Maya pagan el producto en efectivo en base a la calidad de la miel, mientras que Miel Izamal realiza el pago a crédito.

Las grandes empresas comercializadoras referidas previamente tienen sus matrices ubicadas en la ciudad de Mérida, en donde recolectan la miel de los otros centros de acopio con que cuentan en los demás municipios productores de la entidad para finalmente procesarla y venderla al mercado nacional o exportarla hacia otros países del mundo.

Entre las principales estrategias que siguen estas empresas comercializadoras para atraer apicultores hacia sus centros de acopio están: mantener el centro de acopio en las mejores condiciones físicas y de operación, el tener una mejor organización en su proceso administrativo y comercial, y el mejorar el trato que se les da a los apicultores (ofrecer el mejor precio en el mercado). En tanto que entre las estrategias que utilizan para mantener a los apicultores que ya les ofrecen su producto en cada cosecha, se encuentran: el dar créditos a estos, el ofrecer un buen trato al cliente, el dar pláticas de capacitación técnica y el promover la importancia de la producción de miel.

\subsection{Canales de Comercialización de la miel en el municipio de Espita}

El principal canal de comercialización de la miel y de los subproductos de la colmena en el municipio de Espita, Yucatán (Figura 2) está integrado por los siguientes agentes: los productores, los centros de acopio local, las empresas comercializadoras y por último los consumidores, también existe un canal secundario el cual parte del productor y de ah directo al consumidor.

Figura 2

Canales de Comercialización en el Municipio de Espita, Yucatán.

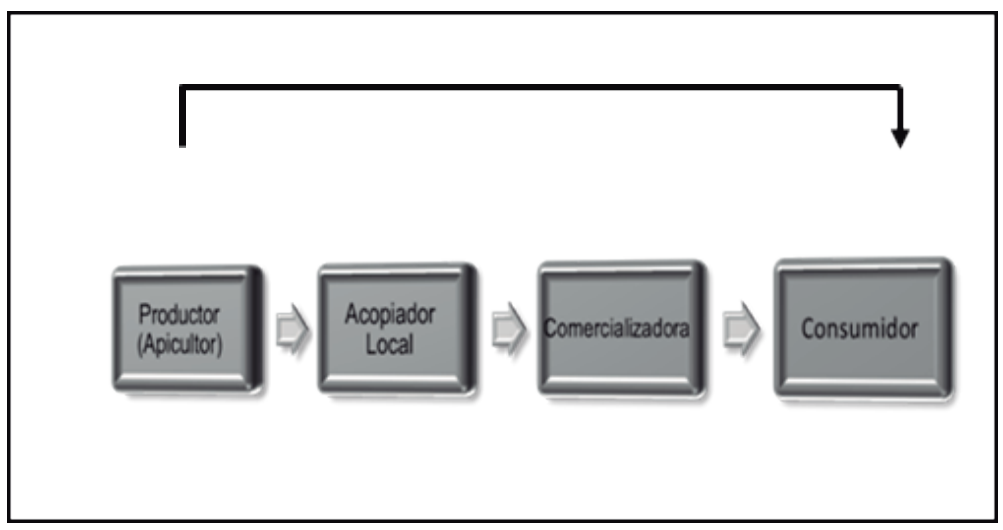

Fuente: Elaborado por el autor con la información de campo.

La figura 3 a continuación muestra el canal principal de distribución de la miel producida en el municipio en forma ampliada. Como se observa, la miel que recolectan los centros de acopio en el canal principal se distribuye en primera instancia al centro de acopio de la ciudad de Valladolid y de ahí, una parte (la mayor) se dirige a la ciudad de Mérida para su procesamiento y colocación final en el mercado internacional. 
En tanto que la otra (la menor) se envasa y comercializa en Valladolid y su región de influencia.

Figura 3

Canal Princinal de Comercialización ampliado de la Miel en Espita, Yucatán.

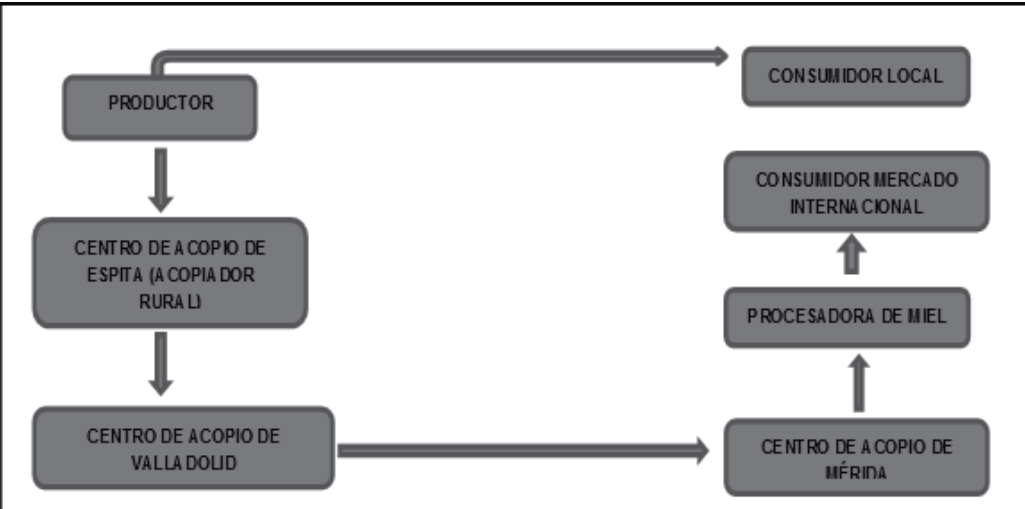

Fuente: Elaborado por el autor con la información de campo.

\subsection{Márgenes Comerciales de los Apicultores de Espita}

Para realizar el cálculo de los márgenes de comercialización, que dependerán de la diferencia entre el precio que pague el agente comercial por el producto que adquiere y el precio que conseguirá que le paguen por la venta, se utilizó el método indirecto propuesto por Caldentey (1992) y la información que se requirió para obtenerlos fueron: el precio por kilogramo que recibe el productor, el precio al mayoreo que paga el acopiador y el precio de venta al consumidor en la municipalidad. Los resultados son los que se presentan a continuación:
Cuadro 3.

\begin{tabular}{|c|c|c|c|c|}
\hline \multicolumn{5}{|c|}{ Márgenes de Comercialización de 1 kilogramo de Miel de Abeja en Espita. } \\
\hline \multirow[b]{2}{*}{ CONCEPTOS } & \multirow[b]{2}{*}{$\begin{array}{c}\text { PRECIO } \\
\text { (1KG.) }\end{array}$} & \multicolumn{2}{|c|}{ MÁRGENES } & \multirow{2}{*}{$\begin{array}{c}\text { PARTICIPACIÓN } \\
\text { RELATIVA }^{2} \\
\%\end{array}$} \\
\hline & & $\begin{array}{c}\text { ABSOLUTO } \\
\$ / 1 \mathrm{~kg}\end{array}$ & $\underset{\%}{\text { RELATIVO }^{1}}$ & \\
\hline Precio de venta del productor & 30 & & & 75 \\
\hline \begin{tabular}{|l|} 
Precio de venta del \\
Acopiador Rural \\
\end{tabular} & 32 & & & \\
\hline $\begin{array}{l}\text { a) Margen del acopiador } \\
\text { rural }\end{array}$ & & 2 & 6.5 & 5 \\
\hline $\begin{array}{l}\text { b) Margen de la empresa } \\
\text { detallista } \\
\text { c) Margen Total }\end{array}$ & & $\begin{array}{l}8 \\
10 \\
\end{array}$ & 26.6 & $\begin{array}{l}20 \\
25 \\
\end{array}$ \\
\hline $\begin{array}{l}\text { Precio de venta al } \\
\text { consumidor local }\end{array}$ & 40 & & & \\
\hline
\end{tabular}

Fuente: Elaborado por el autor con la información de campo.

La interpretación del cuadro anterior indica que los márgenes absolutos de comercialización de $1 \mathrm{~kg}$ de miel en Espita resultaron ser: de dos pesos para el acopiador rural y de ocho pesos para la empresa que vende al detalle en el municipio. Estos márgenes suman diez pesos, el cual es el valor del margen comercial total.

El valor de este margen absoluto tiene una participación relativa en el precio de venta al último agente de este canal comercial del $25 \%$, o sea, que de cada peso pagado a la empresa detallista 25 centavos son retenidos por la intermediación, en tanto que los 75 centavos restantes quedan en manos del productor. Sin embargo, considerando los períodos de tiempo de realización de operaciones, el productor requiere casi de seis meses para obtener esta participación, mientras que la intermediación en un período de días o algunas semanas lo obtiene. 
No se debe olvidar que los apicultores del municipio de Espita son quienes tienen el mayor trabajo y riesgo en el desarrollo productivo de esta actividad, a diferencia de los acopiadores, además de que no todo el año producen miel, la actividad se da por temporadas, y tienen que lidiar año con año con el riesgo que implican los fenómenos climáticos y los cambios que se dan en los precios de la miel en el mercado.

De los agentes comerciales o intermediarios que participan en este canal, la empresa detallista $(26.6 \%$ ) fue quien tuvo la mayor participación en el precio de venta al consumidor local, siguiéndole en importancia el acopiador rural $(6.5 \%)$ de la empresa mayorista 4 .

Esta participación es indicativa, en virtud de que los ingresos y ganancias de cada uno de ellos dependen del volumen de miel manipulada a la semana o al mes, en donde aparentemente la empresa detallista local lleva ventaja, sin embargo, dado que el mayor volumen de la producción de miel en Espita es captada por los acopiadores de las grandes empresas mayoristas del estado, esta ventaja a final de cuentas recae en estas últimas (la cantidad de miel que vende el apicultor directamente al consumidor, así como la cantidad que retiene para autoconsumo, resultaron muy bajas en la municipalidad, de solo un $5 \%$ respectivamente para cada caso).

A lo anterior, se debe agregar que los acopiadores solo se encargan de recolectar, transportar y entregar el producto a la matriz a la cual pertenecen, sin tener que correr altos riesgos ni tener que realizar fuertes inversiones para efectuar su actividad comercial y obtener su ganancia.

\section{CONCLUSIONES}

El municipio de Espita, Yucatán es un municipio productor de miel por tradición, sin embargo, no se le ha dado la importancia debida al desarrollo de esta actividad productiva en el mismo. Entre las razones principales de esto se encuentran: el que la gran mayoría de sus apicultores son personas mayores y el que casi la totalidad de estos llevan a cabo la producción de miel para obtener ingresos adicionales a los de su actividad principal. Por lo tanto, se puede decir que la apicultura en este municipio es una actividad secundaria.

Las principales características que presentan los apicultores de esta localidad son: poseer de una a 50 colmenas, trabajar a nivel familiar, realizar la mayoría de las tareas en su apiario, ubicarse en terrenos ejidales, utilizar poca tecnología o a nivel artesanal, poca inversión en la actividad y no contar con la capacitación técnica necesaria.

Los productos de la colmena que por lo general comercializan los apicultores de Espita son básicamente la miel y la cera, y el transporte de ambos productos hacia el mercado de venta lo realiza el apicultor en vehículos automotrices rentados principalmente.

Las ventas de mayor volumen de miel que realizan al año estos apicultores son en los meses de enero a mayo. En tanto que la forma más generalizada como establecen las relaciones de compra-venta, tanto en miel como en cera, es la que se da cuando el apicultor busca al comprador para realizar la transacción.

El acuerdo de las operaciones comerciales de la venta de miel y cera se finiquitan por lo general a través de la forma de pago de una parte al contado y la otra a crédito. Y en cuanto a la forma en que se establece la venta del producto, en la mayoría de los casos el apicultor es quien busca al comprador para la transacción.
${ }^{4}$ No se presenta el cálculo del margen comercial del canal de comercialización principal en virtud de que no fue posible determinar los precios de venta al por mayor de la miel en el estado.
Enero a Junio de 2012 - Págs: 9-35 
Las empresas comercializadoras con presencia en la municipalidad son sucursales de las grandes compañías que operan en el Estado (Miel Izamal, Miel Mex, Miel Oaxaca y Apícola Maya), las cuales se encargan de recolectar la producción de miel y cera en el municipio a través de sus centros de acopio. Estos centros determinan el precio de compra de la miel que se comercializa mediante el oligopsonio de este producto en el municipio, con lo cual el poder de negociación en la formación del precio al que se vende este en sus manos, y resultan los más beneficiados en el proceso comercial al crear una barrera a los apicultores en el proceso de acopio de miel. De esta forma, el precio a pagar al apicultor, realizado por estos intermediarios en el municipio, responde a un precio previamente fijado y no a las condiciones de la oferta.

En cuanto a los canales de comercialización de la miel, el principal canal en Espita, o el que moviliza la mayor parte de la producción, es corto y está enfocado a la ciudad de Mérida para de ahí colocar el endulzante en el mercado internacional.

Los márgenes absolutos de comercialización evidencian que de los agentes comerciales que participan en el canal de comercialización en el municipio, la empresa detallista es la que tiene la mayor participación en el precio de venta al consumidor local, siguiéndole en importancia el acopiador rural de la empresa mayorista, sin embargo, dado que el mayor volumen de la producción es captada por los acopiadores de las grandes empresas mayoristas del estado, la ventaja en el nivel de ganancia recae en estas últimas.

En general, se puede decir que los principales problemas e ineficiencias detectadas en el proceso de comercialización de los productos apícolas que se obtienen en el municipio de Espita, Yucatán son:

- Falta de dinero suficiente de la venta de sus productos que les permita a los apicultores renovar el equipo y los materiales que la actividad requiere.
- Escasa infraestructura comercial con que cuentan los apicultores.

- Los bajos precios al productor en el mercado y la forma como estos son determinados por parte de las empresas mayoristas. Y

- Desconocimiento del apicultor de estrategias de comercialización de sus productos.

Por último, se puede afirmar que la estructura oligopsónica de los centros de acopio que intervienen en el proceso de comercialización de miel en el municipio de Espita da como consecuencia que el poder de fijación del precio de compra lo tengan estos intermediarios comerciales, y con ello el poder de negociación, y que la comercialización de la miel no sea un proceso dinámico ni beneficioso (ganancia comercial) para el apicultor, este tiene el mayor gasto de recursos, tiempo y trabajo en la producción mientras que los intermediarios solo compran y movilizan en tiempos cortos la miel hacia sus matrices llevándose el mayor margen comercial sin realizar tanto esfuerzo y riesgo. Todo esto evidencia que la relación analizada es desventajosa para el apicultor y refleja que no existe un comercio justo en el municipio. 


\section{REFERENCIAS}

Andrew W. Shepherd. 1995. "Guía para el Cálculo de los Costos de Comercialización”. FAO. Roma.

Caldentey Pedro. 2004. "Comercialización de Productos Agrarios". Editorial agrícola Española, Quinta Edición, Madrid, España.

ASERCA. 2010. "Situación Actual y Perspectiva de la Apicultura en México".Revista Claridades Agropecuarias, número 199, marzo pp 3-34. México DF.

E1 Economista. 2011. "Exportaciones de miel alcanzan cifras récord. Disponible:http://eleconomista.com.mx/industrias/2011/06/12/ex portaciones-miel-alcanzan-cifras-record.

El Financiero. 2009. "Nueva denominación de origen: Miel de la península de Yucatán”. http://iptango.blogspot.com/2010/10/nuevadenomina cion-de-origen-miel-de-la.html

Gobierno del estado de Yucatán. 2010. “Municipios de Yucatán”. En: http://www.yucatan.gob.mx/estado/municipios/ver_municipio .jsp? id=32 Güemes, R.F.J., Echazarreta, C.M. y Villanueva, R. 2002. "Condiciones de la apicultura en Yucatán y del mercado y sus produtos". Disponible en: www.miel.uqroo.mx

Güemes, R.F.J., Echazarreta, C.M. y Villanueva, G.R., Pat F.J.M. y Gómez, A.R. 2003. "La apicultura en la península de Yucatán, actividad de subsistencia en un entorno globalizado". Revista Mexicana de Caribe, No. 16, Vol. VIII, pp.117-132. Universidad de Q. Roo.

Haag M., Herman y Soto José. 1984."E1 Mercado de los Productos Agropecuarios”. Editorial Limusa México D.F. Quinta reimpresión.

Hernández Sampieri, Roberto et al. 2010. "Metodología de la Investigación. McGraw-Hill. Quinta Edición.

MalebranchEraso D. Armando. 2006."Cálculo de los Márgenes de Comercialización”. Gestión de Proyectos y Desarrollo Comunitario. Bogota DC.Colombia. En:www.amalebranch.8m.com/EAEMPIEm6.htm
Magaña Magaña, Miguel Ángel, Aguilar Arrieta, Ana, Lara y Lara, Pedro y Sanginés García, Roberto. 2007.“Caracterización socioeconómica de la act. apícola en el estado de Yucatán”, Agronomía 15 (2), pp. 17-24. México.En: agronomia.ucaldas.edu.co/downloads agronomia15(2) 2.pdf

Medina García, Minneth. 2006."Comercio Justo como alternativa de comercialización: el caso de la miel de exportación de Yucatán”, Tesis, UADY,Facultad de Contaduría y Administración.

Mendoza, V. Gilberto. 1990."Metodología para el Estudio de Canales y Márgenes de Comercialización de Productos agropecuarios", publicada por IICS biblioteca Venezuela.

OCDE. 1981."El análisis de Márgenes de Comercialización de productos Alimentarios, Objetos, Métodos y Utilización”. OCDE. París.

Gobierno del Estado de Yucatán. 2008."Plan municipal de desarrollo rura sustentable de Espita municipio del estado de Yucatán". Programa de Fortalecimiento de los Consejos de Desarrollo Rural Sustentable. Yucatán, México.

SAGARPA. 2009."Servicio de Información Agroalimentaria y Pesquera". México. En: www.campomexicano.gob.mx/portal siap/Integracion/

Secretaria de Fomento Económico. 2010.“Comercio Exterior”. Revista Impulso Económico. No. 10. Gobierno del Estado de Yucatán.

SIAP. 2010 ."Balanza agropecuaria y agroindustrial". En www.campomexicano.gob.mx/portal siap/Integracion/Estadistica Derivada/ComercioExterior/BalanzaComercial/balanzaAV.pdf

Stamer H. 1999.“Teoría del Mercado Agrario”. Edición Academia. León España.

Villanueva, G. R. y Colli-Ucán, W. 1996."La apicultura en la península de Yucatán, México y sus perspectivas”. En: Folia Entomológica Mexicana. No. 97, pp. 55-70. Instituto de Ecología. 